\title{
Struma Ovarii Mimeting Malignant Ovarian Tumor in Preoperative Examinations
}

\section{Gonzalez-Bosquet $\mathbf{E}^{1^{*}}$, Jou Muñoz $\mathrm{C}^{2}$, Sousa Cacheiro $\mathbf{P}^{3}$}

${ }^{1}$ Department of Obstetrics and Gynecology, Sant Joan de Déu University Hospital, Pg. Sant Joan de Déu, 2080950 Esplugues (Barcelona), Spain

${ }^{2}$ Department of Pathology, Sant Joan de Déu University Hospital, Pg. Sant Joan de Déu, 2080950 Esplugues (Barcelona), Spain

${ }^{3}$ Department of Radiology, Sant Joan de Déu University Hospital, Pg. Sant Joan de Déu, 2080950 Esplugues (Barcelona), Spain

*Corresponding Author: Gonzalez-Bosquet E, Department of Obstetrics and Gynecology, Sant Joan de Déu University Hospital, Pg. Sant Joan de Déu, 2080950 Esplugues (Barcelona), Spain; Tel: 932804000; E-mail: edugonzalez@hsjdbcn.org

Received: 22 April 2018; Accepted: 01 June 2018; Published: 04 June 2018

\begin{abstract}
We present the case of a 39 year-old woman with a possible malignant ovarian tumor suspected in imaging tests. After the laparoscopic surgery removal of the ovary, the definitive histological diagnosis is struma ovarii, a rare benign ovarian tumor. We take the case to discuss different aspects of the diagnosis of this rare tumor and how it can be easily confused with an ovarian cancer.
\end{abstract}

Keywords: Ovarian Cancer; Laparoscopic Surgery

\section{Introduction}

Ovarian teratoma tumor originated from monodermal layers of thyroid tissue is defined as struma ovarii [1]. It is a rare benign tumor, accounting for $0.3-1 \%$ of the ovarian tumors and $2-3 \%$ of ovarian teratomas [2-4]. To reach the diagnosis of a struma ovarii, the tumor must either composed in more than $50 \%$ by thyroid component or with less than $50 \%$ but associated with hyperthyroidism symptoms [5]. Other monodermal teratomas are carcinoid and neural tumors.

Preoperative study combines ultrasonography, computer tomography (CT) scan and use of serum biomarkers in an attempt to differentiate malignancy, because struma ovarii is a rare tumor with nonspecific clinical presentation [6]. 
The treatment of struma ovarii is surgical consistent in the removal of the ovarian cyst or mass, to enable histological diagnosis to rule out ovarian malignancy [3].

Surgical approach, laparoscopic versus open surgery, is also controversial due to the size of the tumor and the suspicion of malignancy, because of the risk of the rupture of the tumor and the spread of possible malignant cells. We present a case of struma ovarii managed with laparoscopic surgery.

\section{Case Report}

A 39 year-old female patient referred to our hospital from another center due to an ovarian cyst diagnosed during the management of a spontaneous abortion. Her medical history included an hypothyroidism treated with Thyroxine 50 mg, due to Graves Basedow hyperthyroidism treated with radioactive iodine nine years ago. She used birth control pills for 10 years and was currently looking for a pregnancy, she had no other relevant medical problems.

The cyst measure 130x80 mm and tumor the marker Ca 125 was elevated $(108 \mathrm{u} / \mathrm{ml}$, normal<35). The patient was clinically euthyroid, with a TSH value of $2.99 \mu \mathrm{U} / \mathrm{ml}$.

General examination revealed moderated obesity, and in the vaginal exploration the tumor was difficult to measure as it was situated in Douglas space. Combinated vaginal and abdominal ultrasound was performed confirming the presence of a multilocular and irregular ovarian cyst of $117.5 \mathrm{~mm} \times 86.3 \mathrm{~mm}$ with solid component of more than 50 mm and vascularization (Figure 1 and 2). CT scan observed a pelvis multilocular cystic tumor with two solid components measuring 29mm and $16 \mathrm{~mm}$, positive for contrast and a mild ascites (Figure 3). The results of the requested tumor markers were: CEA $1.1 \mu \mathrm{g} / \mathrm{L}(\mathrm{N}<5)$, Ca 125=108 U/ml $(\mathrm{N}<35)$, Ca $19.9=19 \mathrm{U} / \mathrm{ml}(\mathrm{N}<39)$ and $\mathrm{HE} 4=31.3 \mathrm{pmol} / \mathrm{L}(\mathrm{N}<70)$.

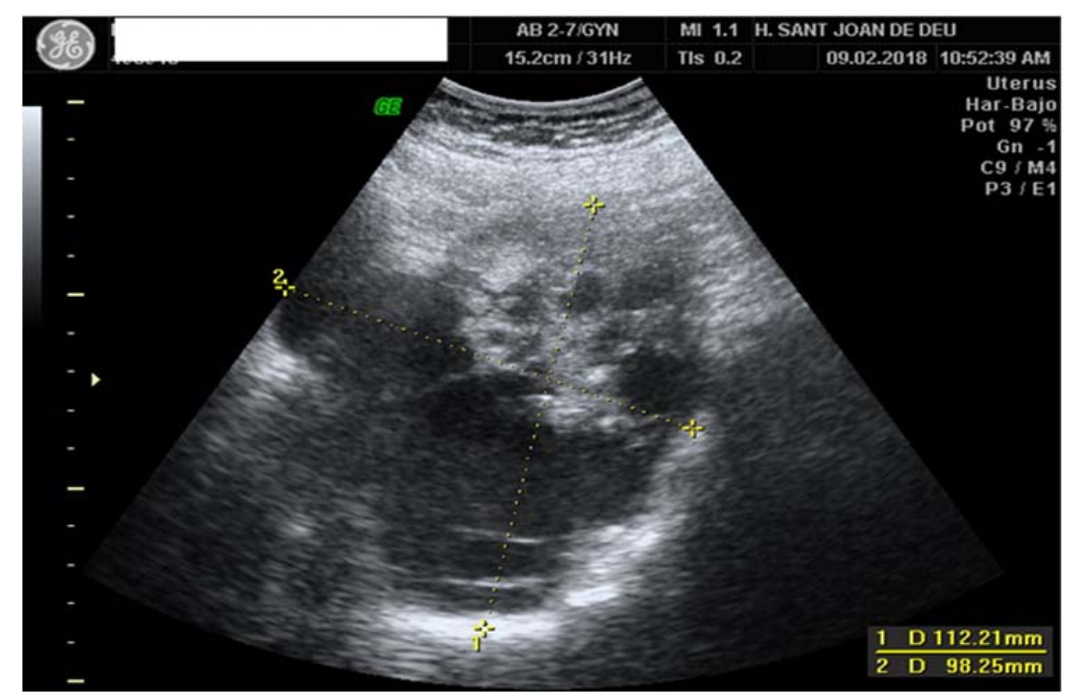

Figure 1: Abdominal ultrasound. Ovarian cyst of 112 x $98 \mathrm{~mm}$ of size, with solid component of $55 \mathrm{~mm}$. 


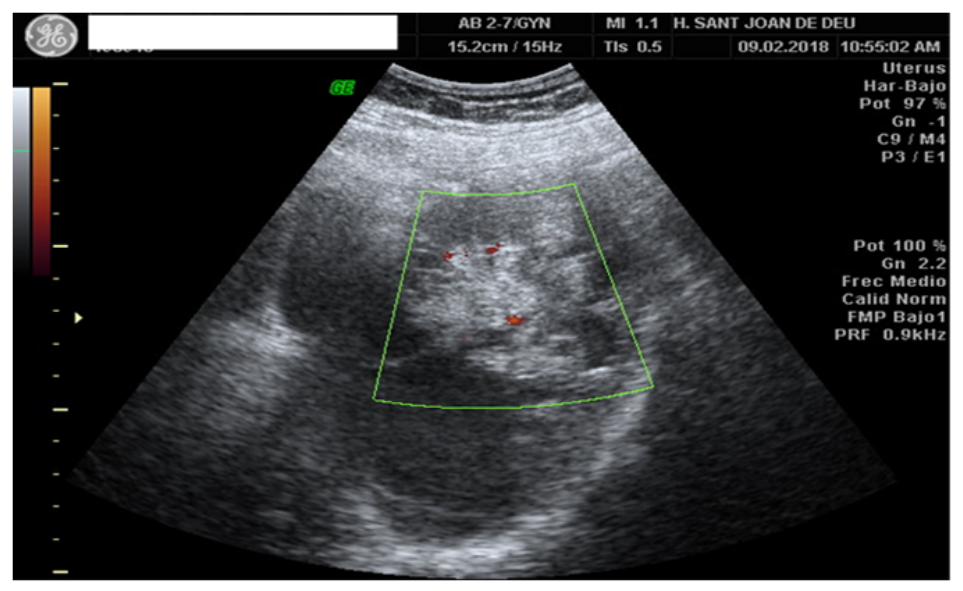

Figure 2: Abdominal ultrasound. Doppler vascularization of solid part of the tumor

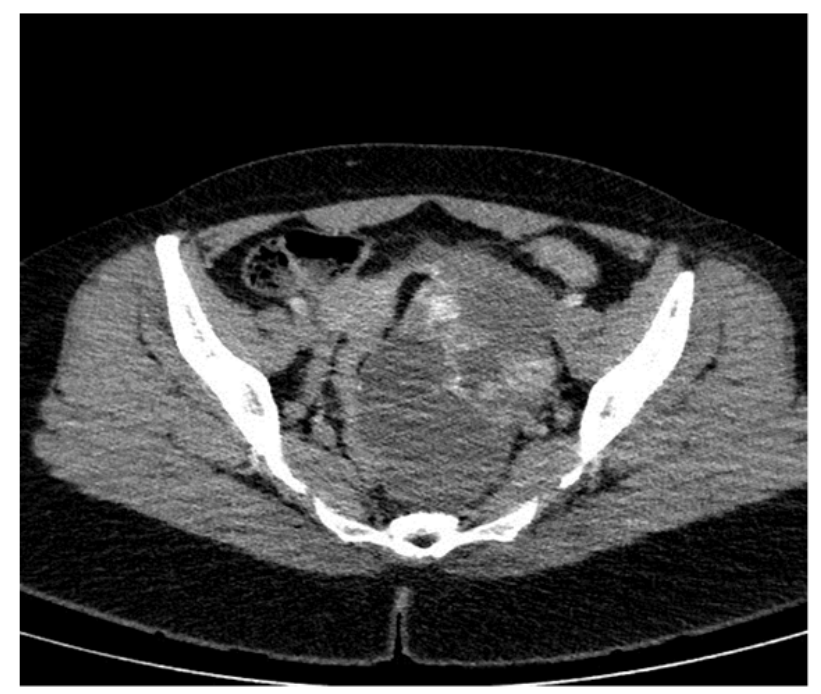

Figure 3: CT scan of the ovarian heterogeneous tumor (cystic and solid) with mild ascites

Although following IOTA simple rules the ovarian cyst was very suspicious of malignant ovarian tumor, normal HE4 and mild elevation of Ca 125, in patient with ascites, raised doubts about the malignancy of the tumor. So a laparoscopic approach was perform in order to rule out a histological diagnosis with deferred análisis of the ovary, in order to give a better information for the patient in case of malignancy.

An open laparoscopy technique (Hasson) was used due to the size of the tumor. A minimum amount of ascitis was found in the pelvis and aspirated for analysis. No evidence of tumor spreads were found in the upper abdomen or pelvis and the uterus and right adnexa were normal. A multilocular cyst measuring $130 \mathrm{~mm}$ was find in the right anexa, with not clear evidence of malignancy. A right laparoscopic anexectomy without problems were performed, the tumor was extracted with an endobag of $150 \mathrm{~mm}$. During the extraction of the adnexe, extravasation of the cystic 
content to the pelvis was detected. Postoperatory recovery was correct and the patient was discharged less than 24 hours after the surgery.

The final histologic diagnosis was struma ovarii, 100\% of the tumor was composed by thyroid tissue, malignancy was ruled out in the tumor and ascitic liquid..

\section{Discussion}

Struma ovarii usually presents in the fifth decade of live [6]. It is a rare benign tumor that could present symptoms of Hyperthyroidism in 5-8\% of the cases, our patient had prior medical history of hyperthyroidism due to Graves Basedow nine years before no related with the current tumor. These tumors can also manifest pseudo-Meigs syndrome [7] as in our case which presents with a mild ascites.

Preoperative study includes ultrasound, CT scan and tumor markers. In a study [8], which include 68 cases of struma ovarii $65 \%$ had a preoperative diagnosis of possible ovarian malignancy, as in our case. Preoperative differential diagnosis can be difficult even for experienced oncologist, because echo patterns mimic ovarian cancer. Mild elevation of Ca 125 is very frequent due to peritoneal irritation of ascitis ( Meigs Syndrome of struma ovarii) which it is also seen in ovarian cancer but with higher Ca 125 values and more severe ascites and pleural effusion.

HE4 is a more specific tumor marker because is not affected by the ascites and in most of the cases of struma ovarii [6], like in ours, is negative $(<70)$.

CT scan, it is not very usefull to rule out malignancy because struma ovarii usually presents as heterogeneous cysts with solid component and ascites us in our case.

Magnetic resonance (MRI) could demonstrate low signal observed for the mass on T2W1, occasionally referred to the vacuum phenomenon, is associated with the viscous fluid within cysts, which is a specific indicator of struma ovarii [9].

The clinical presentation of these ovarian tumors lead to a high percentage of open surgical approach, in large series, 73.5\% [8], because the risk of malignancy.

However, a review by Ezon et al reported that the use of the laparoscopic approach did not negatively impact disease-free or overall survival rates [10].

Treatment of benign struma ovarii includes cystectomy and salpingo-oophorectomy depending of desire for future fertility, in our case not normal ovary tissue was identify and the suspicious of malignancy led us to perform a salpingo-ooforectomy. Data on recurrence of benign struma ovarii is scarce [11]. 
In conclusion struma ovarii is a rare ovarian benign tumor which could mimic an ovarian cancer because the results preoperatory study ( ultrasound, CT scan and Ca 125). HE4 could help differentiate it from a malignant tumor.

Treatment of these tumor includes cystectomy or salpingo-oophorectomy, and does not require prolonged follow up periods or excessive postoperative investigation unless women are symptomatic.

\section{References}

1. D’Antonio A, Caleo, Caleo O, et al. Hashimoto thyroiditis as a manifestation of struma ovarii. Endocrinologist 20 (2010): 220-221.

2. Outwater EK, Siegelman ES and Huntn JL. Ovarian teratomes: tumor types and imaginin characteristics. Radiographics 21 (2001): 475-490.

3. Yoo SC, Chang KH, Lyu MO et al. Clinical characteristics of struma ovarii. J Gynecol Oncol 19 (2008): 135-138.

4. Zhu Y, Wang C, Zhang GN et al. Papillary thyroid càncer located in malignant struma ovari with omentum metàstasis: A case report and review of the literatura. World J Surg Oncol 14 (2016): 17.

5. Krisnamurthy A, Ramshankar V, Vaidyalingam V and Majhi U. Synchronous papillary carcinoma thyroid with malignant struma ovarii: A management dilema. Indian J Nucl Med 28 (2013): 243-245.

6. Diavatis S, Papanikolaou A. Level of HE4 is correlated with diagnosis of struma ovarii: A case report. Am J Case Rep 17 (2016): 459-461.

7. Ali Kemal Sivrioglu, Muzaffer Saglam, Guner Sonmez, Mehmet Deveer. Pseudo-Meigs'syndrome associated with struma ovarii. BMJ Case Report 2013.

8. Wee JYS, Li Xinyi, Chern BSM, Chua ISY. Struma ovarii: management and follow-up of a rare ovarian tumour. Singapure Med J 56 (2015): 35-39.

9. Okada S, Ohaki Y, Kawamura T et al. Cystic struma ovarii: imaginin findings. J Comput Assist Tomogr 24 (2000): 413-415.

10. Ezon I, Zilbert N, Pinkney L et al. A large struma ovarii tumor removed via laparoscopy in a 16-year-old adolescent. J Pediatr Surg 42 (2007): E19-E22.

11. Ayhan A, Yanik F, Tuncer R, Tuncer ZS, Ruacan S. Struma ovarii. Int J Gynaecol Obstet 42 (1993): 143176.

Citation: Gonzalez-Bosquet E, Jou Muñoz C, Sousa Cacheiro P. Struma Ovarii Mimeting Malignant Ovarian Tumor in Preoperative Examinations. Obstetrics and Gynecology Research 1 (2018): 075-079.

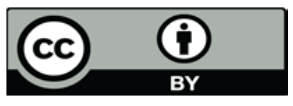

This article is an open access article distributed under the terms and conditions of the

Creative Commons Attribution (CC-BY) license 4.0 